九州大学学術情報リポジトリ

Kyushu University Institutional Repository

乳腺造影ダイナミックMR I における背景乳腺の増 強と乳癌の検出能に対する月経周期の影響 : アジア 人での多施設研究

神谷，武志

https://doi.org/10.15017/2348721

出版情報：Kyushu University，2019，博士（医学），論文博士 バージョン：

権利関係 : 
Research article

\section{Effects of menstrual cycle on background parenchymal enhancement and detectability of breast cancer on dynamic contrast-enhanced breast MRI: A multicenter study of an Asian population}

Takeshi Kamitani $^{\mathrm{a}, *}$, Hidetake Yabuuchi ${ }^{\mathrm{b}}$, Yoshihide Kanemaki ${ }^{\mathrm{c}}$, Mitsuhiro Tozaki ${ }^{\mathrm{d}}$, Tetsuo Sonomura ${ }^{\mathrm{e}}$, Waka Mizukoshi ${ }^{\mathrm{f}}$, Waka Nakata ${ }^{\mathrm{g}}$, Taro Shimono ${ }^{\mathrm{h}}$, Misugi Urano ${ }^{\mathrm{i}}$, Toshiko Yamano $^{j}$, Fumi Kato ${ }^{\mathrm{k}}$, Megumi Kuchiki ${ }^{1}$, Nobuyuki Shiragami ${ }^{\mathrm{m}}$, Hisami Yanagita ${ }^{\mathrm{n}}$, Eisuke Katsuda $^{\circ}$, Masako Kataoka ${ }^{\mathrm{p}}$, Ken Yamaguchi ${ }^{\mathrm{q}}$, Takuro Horikoshi ${ }^{\mathrm{r}}$, Tatsuya Gomi ${ }^{\mathrm{s}}$, Miwako Nozaki $^{\mathrm{t}}$, Motoi Shiotani ${ }^{\mathrm{u}}$, Maki Amano ${ }^{\mathrm{v}}$, Hirokazu Saigusa ${ }^{\mathrm{w}}$, Shunichi Sadaoka ${ }^{\mathrm{x}}$, Hisashi Kamiya $^{\mathrm{y}}$, Makoto Kubo ${ }^{\mathrm{z}}$, Nami Yamashita ${ }^{\mathrm{A}}$, Hidetaka Yamamoto ${ }^{\mathrm{B}}$, Hiroshi Honda ${ }^{\mathrm{a}}$

${ }^{a}$ Department of Clinical Radiology, Graduate School of Medical Sciences, Kyushu University, 3-1-1 Maidashi, Higashi-ku, Fukuoka, 812-8582, Japan

${ }^{\mathrm{b}}$ Department of Health Sciences, Graduate School of Medical Sciences, Kyushu University, 3-1-1 Maidashi, Higashi-ku, Fukuoka, 812-8582, Japan

${ }^{\mathrm{c}}$ Department of Radiology, St. Marianna University School of Medicine, 2-16-1 Sugao, Miyamae-ku, Kawasaki, Kanagawa, 216-8511, Japan

${ }^{\mathrm{d}}$ Department of Radiology, Sagara Hospital Affiliated Breast Center, 3-28 Tenokuchi-cho, Kagoshima 892-0845, Japan

e Department of Radiology, Wakayama Medical University, 811-1 Kimiidera, Wakayama, 641-8509, Japan

${ }^{\mathrm{f}}$ Department of Diagnostic Radiology, Saitama Medical University International Medical Center, 1397-1 Yamane, Hidaka, Saitama, 350-1298, Japan

${ }^{\mathrm{g}}$ Department of Radiology, Jichi Medical University School of Medicine, 3311-1 Yakushiji; Tochigi, Shimotsuke, 329-0498, Japan

${ }^{\mathrm{h}}$ Department of Diagnostic and Interventional Radiology, Osaka City University Graduate School of Medicine, 3-3-138 Sugimoto, Sumiyoshi-ku, Osaka, 558-8585, Japan

i Department of Radiology, Nagoya City University Graduate School of Medical Sciences and Medical School, 1 Kawasumi, Mizuho-cho, Mizuho-ku, Nagoya, Aichi, 4678601, Japan

${ }^{\mathrm{j}}$ Department of Radiology, Hyogo College of Medicine, 1-1 Mukogawa-cho, Nishinomiya, Hyogo, 663-8131, Japan

${ }^{\mathrm{k}}$ Department of Diagnostic and Interventional Radiology, Hokkaido University Hospital, Kita 14, Nishi 5, Kita-ku, Sapporo, Hokkaido, 060-8648, Japan

${ }^{1}$ Department of Diagnostic Radiology, Yamagata University Faculty of Medicine, 2-2-2 Iida-Nishi, Yamagata, 990-9585, Japan

${ }^{\mathrm{m}}$ Department of Radiology, Toho University Medical Center Omori Hospital, 6-11-1 Omori-nishi, Ota-ku, Tokyo, 143-8541, Japan

n Department of Radiology, Saitama Medical Center, Saitama Medical University, 1981 Kamoda, Kawagoe, Saitama, 350-8550, Japan

${ }^{\circ}$ Department of Radiology, Aichi Medical University, 1-1 Yazakokarimata, Nagakute, Aichi, 480-1195, Japan

${ }^{\mathrm{p}}$ Department of Diagnostic Imaging and Nuclear Medicine, Kyoto University Graduate School of Medicine, Yoshida-Konoe-cho, Sakyo-ku, Kyoto, 606-8501, Japan

${ }^{\mathrm{q}}$ Department of Radiology, Faculty of Medicine, Saga University, 5-1-1 Nabeshima, Saga, 849-8501, Japan

${ }^{\mathrm{r}}$ Department of Radiology, Chiba University Hospital, 1-8-1 Inohara, Chiba, 260-8677, Japan

${ }^{\mathrm{s}}$ Department of Radiology, Toho University Medical Center Ohashi Hospital, 5-21-16, Omorinishi, Ota-ku, Tokyo, 143-8540, Japan

${ }^{\mathrm{t}}$ Department of Radiology, Dokkyo Medical University Saitama Medical Center, 2-1-50 Minamikoshigaya, Koshigata, Saitama, 343-8555, Japan

${ }^{\mathrm{u}}$ Department of Radiology and Radiation Oncology, Niigata University Graduate School of Medical and Dental Sciences, 757 Ichibancho, Asahimachi-dori, Chuo-ku, Niigata, 951-8510, Japan

${ }^{v}$ Department of Radiology, Juntendo University Nerima Hospital, 3-1-10 Takanodai, Nerima-ku, Tokyo, 177-8521, Japan

${ }^{\mathrm{w}}$ Department of Radiology, The 3rd Hospital of The Jikei University School of Medicine, 4-11-1 Izumihoncho, Komae, Tokyo, 206-8601, Japan

${ }^{\mathrm{x}}$ Department of Radiology, The Jikei University Kashiwa Hospital, 163-1 Kashiwashita, Kashiwa, Chiba, 277-8567, Japan

${ }^{\mathrm{y}}$ Department of Radiology, Granduate School of Medical Science, University of the Ryukyus, 207 Uehara, Nishiharacho, Nakagami, Okinawa, 903-0215, Japan

${ }^{\mathrm{z}}$ Department of Surgery and Oncology, Graduate School of Medical Sciences, Kyushu University, 3-1-1 Maidashi, Higashi-ku, Fukuoka, 812-8582, Japan

A Department of Surgery and Science, Graduate School of Medical Sciences, Kyushu University, 3-1-1 Maidashi, Higashi-ku, Fukuoka, 812-8582, Japan

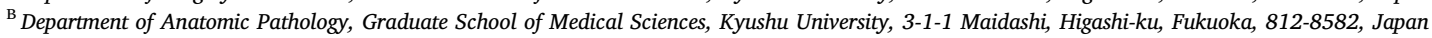

\section{A R T I C L E I N F O}

\section{Keywords:}

Breast

MRI

Menstrual cycle

Background parenchymal enhancement

\begin{abstract}
A B S T R A C T
Purpose: To evaluate the effect of the menstrual cycle on BPE and cancer detectability in an Asian population. Material and methods: 266 premenopausal patients with regular menstrual cycles from 24 centers were included, and 176 of them were diagnosed by pathology as having breast cancer. Thirty-five patients were examined in the menstrual phase (days 1-4), 105 in the proliferative phase (days 5-14), and 126 in the secretory phase (days 15-30).
\end{abstract}

Measurement of the following signal intensities (SIs) were obtained: breast tissue on the unaffected side on a

\footnotetext{
* Corresponding author.

E-mail address: kamitani@radiol.med.kyushu-u.ac.jp (T. Kamitani).
} 
pre-contrast image $\left(\mathrm{SI}_{1}\right)$ and an early-phase image $\left(\mathrm{SI}_{2}\right)$; the SIs of breast tissue on the affected side on a precontrast image $\left(\mathrm{SI}_{3}\right)$ and an early-phase image $\left(\mathrm{SI}_{4}\right)$; and the SIs of breast cancer on a pre-contrast image $\left(\mathrm{SI}_{5}\right)$ and an early-phase image $\left(\mathrm{SI}_{6}\right)$. We calculated the BPE ratio, i.e., $\left(\mathrm{SI}_{2}-\mathrm{SI}_{1}\right) / \mathrm{SI}_{1}$ and the cancer/background enhancement ratio $(\mathrm{C} / \mathrm{B})$ ratio, i.e., $\left(\mathrm{SI}_{6}-\mathrm{SI}_{5}\right) /\left(\mathrm{SI}_{4}-\mathrm{SI}_{3}\right)$. The BPE was classified as minimal, mild, moderate, or marked, and the cancer detectability was classified as excellent, good, or poor independently by two radiologists. Results: The average C/B ratio was 20.1, 15.7, and 9.1 at the menstrual, proliferative, and secretory phases ( $\mathrm{p}<0.001$ ). BPE was determined as moderate or marked in $0 \%$ and $5.4 \%$ at the menstrual phase, $10.3 \%$ and $11.0 \%$ at the proliferative phase, and $17.5 \%$ and $21.7 \%$ at the secretory phase by the two observers, respectively ( $\mathrm{p}=0.01, \mathrm{p}=0.01$ ). The detectability of breast cancer was classified as poor in $0 \%$ and $0 \%, 1.4 \%$ and $13.0 \%$, and $8.0 \%$ and $22.1 \%$ at the menstrual, proliferative, and secretory phases by the two observers, respectively $(\mathrm{p}=0.07, \mathrm{p}=0.02)$.

Conclusion: The menstrual phase and the proliferative phase seem to be suitable for breast MRI of Asian women.

\section{Introduction}

One of the most important roles of breast magnetic resonance imaging (MRI) is the preoperative estimation of the extent of ductal spread of breast cancer, which affects the choice of surgical procedure. A preoperative oversight or underestimation of ductal spread in a breast-conserving surgery presents a great risk of a remaining positive margin that can cause local recurrence. An accurate preoperative evaluation of ductal spread is thus clinically important. Mammography, ultrasound, and MRI have been reported to provide sensitivity of $21 \%-55 \%, 21 \%-89 \%$, and $67 \%-93 \%$, specificity of $86 \%-100 \%$, $76 \%-86 \%$, and $60 \%-90 \%$, and accuracy of $42 \%-72 \%, 50 \%-85 \%$, and $66 \%-92 \%$ for ductal spread [1-4]. MRI has the highest diagnostic performance for ductal spread.

Background parenchymal enhancement (BPE) on contrast-enhanced breast MRI is known to be associated with the menstrual cycle, because breast tissue is influenced by the cyclic hormonal changes - especially estrogen -that occur over the menstrual cycle [5-7]. Estrogen is known to cause the vascularization of breast parenchyma and a proliferation of ductal-acini epithelia, and histamine-like effects such as vasodilation and increased permeability of vessels $[1,8,9]$. Prominent BPE could decrease the diagnostic performance of MRI, because it could disturb lesion detection $[8,10]$. The enhancement of normal breast tissue could result in false-positive findings or even mask a small abnormal enhancing cancer, resulting in a false-negative report [5]. It is thus important to perform breast MRI at the appropriate phase of the menstrual cycle in premenopausal women.

The guidelines from the European Society of Breast Imaging state that the optimal time to perform a breast MRI in a premenopausal women is between the 5th and 12th day after the start of her menstrual cycle [6], but this guideline is based mostly on the results of studies of Western populations [11-13]. To our knowledge, there is only one prior corresponding study of an Asian population, and it was a single-center investigation [14]. In addition, although BPE changes in accord with the menstrual cycle have been described, the effect of BPE on cancer detectability has not been evaluated. We conducted the present study to evaluate the effects of the menstrual cycle on BPE and cancer detectability on dynamic contrast-enhanced breast MRI in an Asian population.

\section{Material and methods}

\subsection{Patients}

We conducted a multicenter prospective study involving 24 hospitals in Japan. The study was approved by the Ethical Review Board on Clinical Studies at each institution. Written informed consent for participation was obtained from all patients. The inclusion criteria were as follows: (1) female patients who underwent dynamic contrast-enhanced breast MRI for a close examination; (2) age 20-69 years; (3) the patient's menstrual cycle information was obtained by interview. First, between November 2013 and March 2015, 962 patients from the 24 centers were enrolled. The exclusion criteria were as follows: (1) patients with a previous history of treatment for breast cancer; (2) the patient's menstrual cycle within the past 3 months was irregular; (3) a histological diagnosis could not be obtained. A final total of 293 premenopausal patients (age range 23-54 years, mean 42.4 years) were included in our analyses, and the breast cancer diagnosis of 183 of these patients was based on pathology examination. Thirty-seven patients were examined in the menstrual phase (days 1-4), 118 in the proliferative phase (days 5-14), and 138 in the secretory phase (days 15-30).

\subsection{MRI protocols}

MRI examinations were performed with a 1.5 or $3 \mathrm{~T}$ system. Axial or coronal views of 3D fast GRE T1-weighted images with fat suppression of bilateral whole breasts with the patients in the prone position were required. No regulation was set regarding the repetition time (TR), echo time (TE), or flip angle (FA). The slice thickness was prescribed to 1-2 mm. Images before and 1-2 min after the administration of contrast medium were used for the evaluation.

\subsection{Image analysis}

\subsubsection{Evaluation 1: BPE of breast tissue on the unaffected side}

For a quantitative assessment, a region of interest (ROI) was set manually to include all of the fibroglandular breast tissue on the unaffected side by one radiologist. Visible cystic areas, fatty tissue, and nodular enhancing areas were avoided. The signal intensities (SIs) of breast tissue on a pre-contrast image $\left(\mathrm{SI}_{1}\right)$ and those on an early-phase image $\left(\mathrm{SI}_{2}\right)$ were measured. We then calculated the BPE ratio for each patient: $\left(\mathrm{SI}_{2}-\mathrm{SI}_{1}\right) / \mathrm{SI}_{1}$. For the qualitative assessment, the BPE results were classified independently by two radiologists (27 and 23 years' experience in breast radiology) as follows: Minimal: $\leq 25 \%$ enhancement of glandular tissue. Mild: $>25 \%$ to $\leq 50 \%$ enhancement of glandular tissue. Moderate: $>50 \%-75 \%$ enhancement of glandular tissue. Marked: $>75 \%$ enhancement of glandular tissue.

\subsubsection{Evaluation 2: cancer detectability}

For the quantitative assessment, we measured the SIs of fibroglandular breast tissue on the affected side on a pre-contrast image $\left(\mathrm{SI}_{3}\right)$ and an early-phase image $\left(\mathrm{SI}_{4}\right)$, and the SIs of breast cancer on a pre-contrast image $\left(\mathrm{SI}_{5}\right)$ and those on an early-phase image $\left(\mathrm{SI}_{6}\right)$. We then calculated the cancer/background enhancement (C/B) ratio for each patient: $\left(\mathrm{SI}_{6}-\mathrm{SI}_{5}\right) /\left(\mathrm{SI}_{4}-\mathrm{SI}_{3}\right)$. For the qualitative assessment, the cancer detectability was classified independently by the above-mentioned radiologists as follows. Excellent: little BPE and easy to detect. Good: weak BPE but detectable. Poor: strong BPE and difficult to detect. 


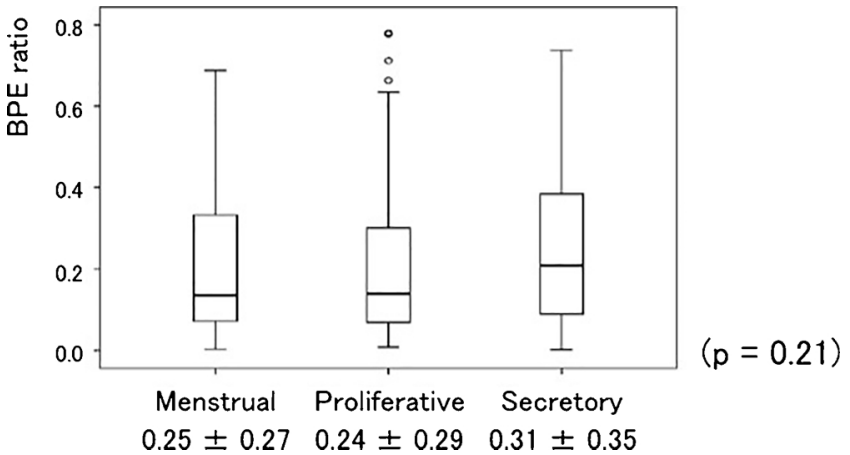

Fig. 1. The BPE ratios. The BPE ratios were $0.25 \pm 0.27$ at the menstrual phase, $0.24 \pm 0.29$ at the proliferative phase, and $0.31 \pm 0.35$ at the secretory phase: $=$ non- significant differences; $\mathrm{p}=0.21$.

\subsection{Statistical analyses}

We evaluated the inter-observer agreements in the qualitative analyses of the BPE classification and cancer detectability by using weighted $\mathrm{k}$ statistics. We compared the results of the quantitative assessment (BPE ratio and C/B ratio) with the menstrual cycles using the Kruskal-Wallis test and the Steel-Dwass test for multiple comparisons. For the qualitative assessment, we compared the proportion of minimalto-mild BPE and that of excellent-to-good cancer detectability with the menstrual cycle by the $X^{2}$ test. P-values $<0.05$ were considered significant. The statistical analyses were performed using SPSS Statistics ver. 21 (IBM, Chicago, IL), R version 3. 2.3 (http://www.r-project.org), or Statcel (OMS, Tokorozawa, Japan).

\section{Results}

\subsection{Inter-observer agreements}

The inter-observer agreements in the qualitative analyses of the BPE classification and cancer detectability were in the moderate range: BPE classification, 0.57 ; cancer detectability, 0.52 .

\subsubsection{Evaluation 1: BPE of breast tissue on the unaffected side}

The BPE ratio averages and standard deviations (SDs) were $0.25 \pm 0.27$ at the menstrual phase, $0.24 \pm 0.29$ at the proliferative phase, and $0.31 \pm 0.35$ at the secretory phase (Fig. 1). There was no significant difference in BPE ratio among the three menstrual phases $(\mathrm{p}=0.21)$.

As summarized in Table 1 , Observer 1 classified 18 cases as minimal, 19 cases as mild, and no case as moderate or marked at the menstrual phase; 65 cases as minimal, 40 cases as mild, 9 cases as moderate, and 3 cases as marked at the proliferative phase; 60 cases as minimal, 53 cases as mild, 23 cases as moderate, and 1 case as marked at the secretory phase. Observer 2 classified 26 cases as minimal, 9 cases as mild, 2 cases as moderate, and no case as marked at the menstrual phase; 71 cases as minimal, 34 cases as mild, 12 cases as

Table 1

Qualitative assessment of BPE by Observer 1 .

\begin{tabular}{llll}
\hline & Menstrual & Proliferative & Secretory \\
\hline $\begin{array}{l}\text { Minimal } \\
\text { or Mild }\end{array}$ & 37 & 105 & 113 \\
$\begin{array}{c}\text { Moderate } \\
\text { or Marked }\end{array}$ & 0 & 12 & 24 \\
\hline
\end{tabular}

$(\mathrm{p}=0.01)$.
Table 2

Qualitative assessment of BPE by Observer 2 .

\begin{tabular}{llll}
\hline & Menstrual & Proliferative & Secretory \\
\hline $\begin{array}{c}\text { Minimal } \\
\text { or Mild }\end{array}$ & 35 & 105 & 118 \\
$\begin{array}{c}\text { Moderate } \\
\text { or Marked }\end{array}$ & 2 & 13 & 30 \\
\hline
\end{tabular}

$(\mathrm{p}=0.01)$

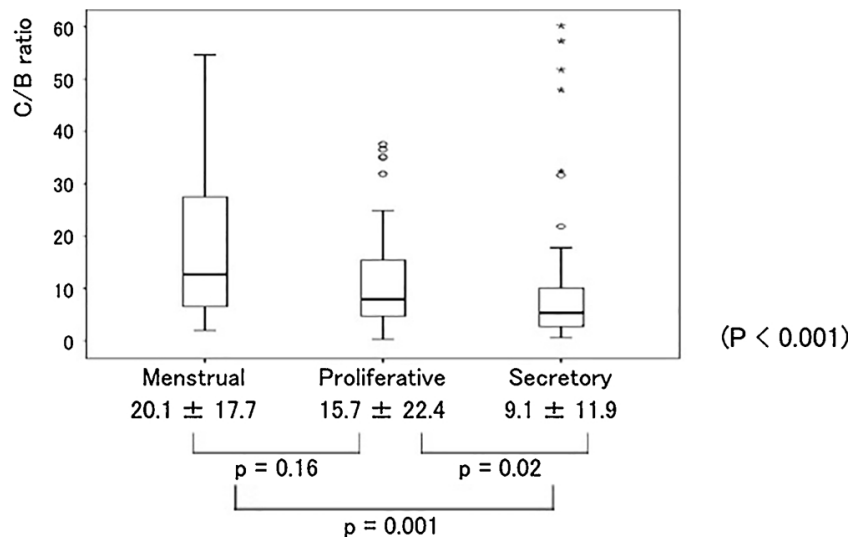

Fig. 2. The C/B ratios. The C/B ratios were $20.1 \pm 17.7$ at the menstrual phase, $15.7 \pm 22.4$ at the proliferative phase, and $9.1 \pm 11.9$ at the secretory phase $(\mathrm{p}<0.001)$. The secretory phase showed the worst C/B ratio.

moderate, and 1 case as marked at the proliferative phase; 76 cases as minimal, 32 cases as mild, 26 cases as moderate, and 4 cases as marked at the secretory phase (Table 2). Thus, BPE was determined as moderate or marked in $0 \%$ and $5.4 \%$ at the menstrual phase, $10.3 \%$ and $11.0 \%$ at the proliferative phase, and $17.5 \%$ and $21.7 \%$ at the secretory phase by the two observers, respectively $(\mathrm{p}=0.01, \mathrm{p}=0.01)$.

\subsubsection{Evaluation 2: cancer detectability}

The $\mathrm{C} / \mathrm{B}$ ratio averages and SDs were $20.1 \pm 17.7$ at the menstrual phase, $15.7 \pm 22.4$ at the proliferative phase, and $9.1 \pm 11.9$ at the secretory phase ( $\mathrm{p}<0.001$ ) (Fig. 2). There were significant differences between the menstrual and secretory phases $(\mathrm{p}=0.001)$, and between the proliferative and secretory phases $(\mathrm{p}=0.02)$. There was no significant difference between the menstrual and proliferative phases $(\mathrm{p}=0.16)$.

As shown in Table 3, Observer 1 classified 24 cases as excellent, 3 cases as good, and no case as poor at the menstrual phase; 64 cases as excellent, 4 cases as good, and 1 case as poor at the proliferative phase; 65 cases as excellent, 15 cases as good, and 7 cases as poor at the secretory phase. Observer 2 classified 23 cases as excellent, 4 cases as good, and no case as poor at the menstrual phase; 48 cases as excellent, 12 cases as good, and 9 cases as poor at the proliferative phase; 48 cases as excellent, 22 cases as good, and 17 cases as poor at the secretory phase (Table 4). Thus, the detectability of breast cancer was classified as poor in $0 \%$ and $0 \%$ at the menstrual phase, $1.4 \%$ and $13.0 \%$ at the proliferative phase, and $8.0 \%$ and $22.1 \%$ at the secretory phase by the

Table 3

Qualitative assessment of cancer detectability by Observer 1 .

\begin{tabular}{llll}
\hline & Menstrual & Proliferative & Secretory \\
\hline $\begin{array}{l}\text { Excellent } \\
\text { or Good }\end{array}$ & 27 & 68 & 80 \\
Poor & 0 & 1 & 7
\end{tabular}

( $p=0.07)$. 
Table 4

Qualitative assessment of cancer detectability by Observer 2 .

\begin{tabular}{llll}
\hline & Menstrual & Proliferative & Secretory \\
\hline $\begin{array}{l}\text { Excellent } \\
\text { or Good }\end{array}$ & 27 & 60 & 70 \\
Poor & 0 & 9 & 17 \\
\hline
\end{tabular}

$(\mathrm{p}=0.04)$. two observers, respectively $(\mathrm{p}=0.07, \mathrm{p}=0.02)$. Representative cases are shown in Figs. 3-5.

\section{Discussion}

The suitability of the proliferative phase for breast MRI was demonstrated in previous investigations. Kuhl et al. described that an enhancement of breast parenchyma occurred during all phases of the menstrual cycle, especially during weeks 1 and 4 , and that the

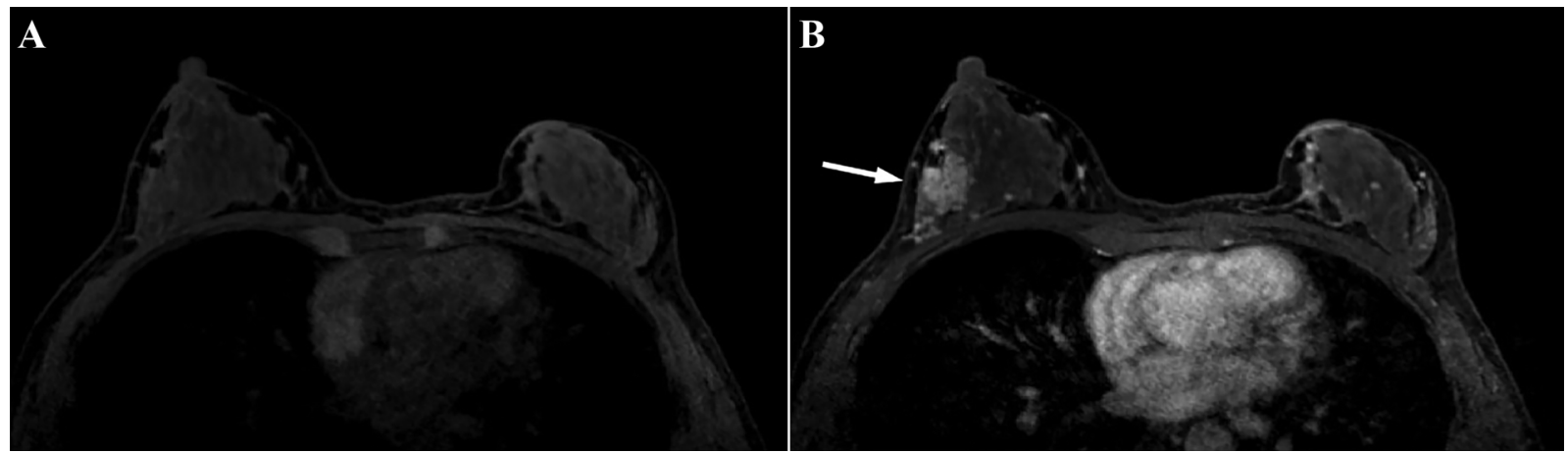

Fig. 3. A 41-year-old female with invasive ductal carcinoma at the menstrual phase.

An axial (a) pre-contrast and (b) post-contrast image clearly demonstrates an enhancing lesion in the outer area of the right breast (arrow). The BPE ratio was 0.06, and both observers classified the BPE as mild. The C/B ratio was 13.5 and qualitative cancer detectability was classified as excellent by both observers.
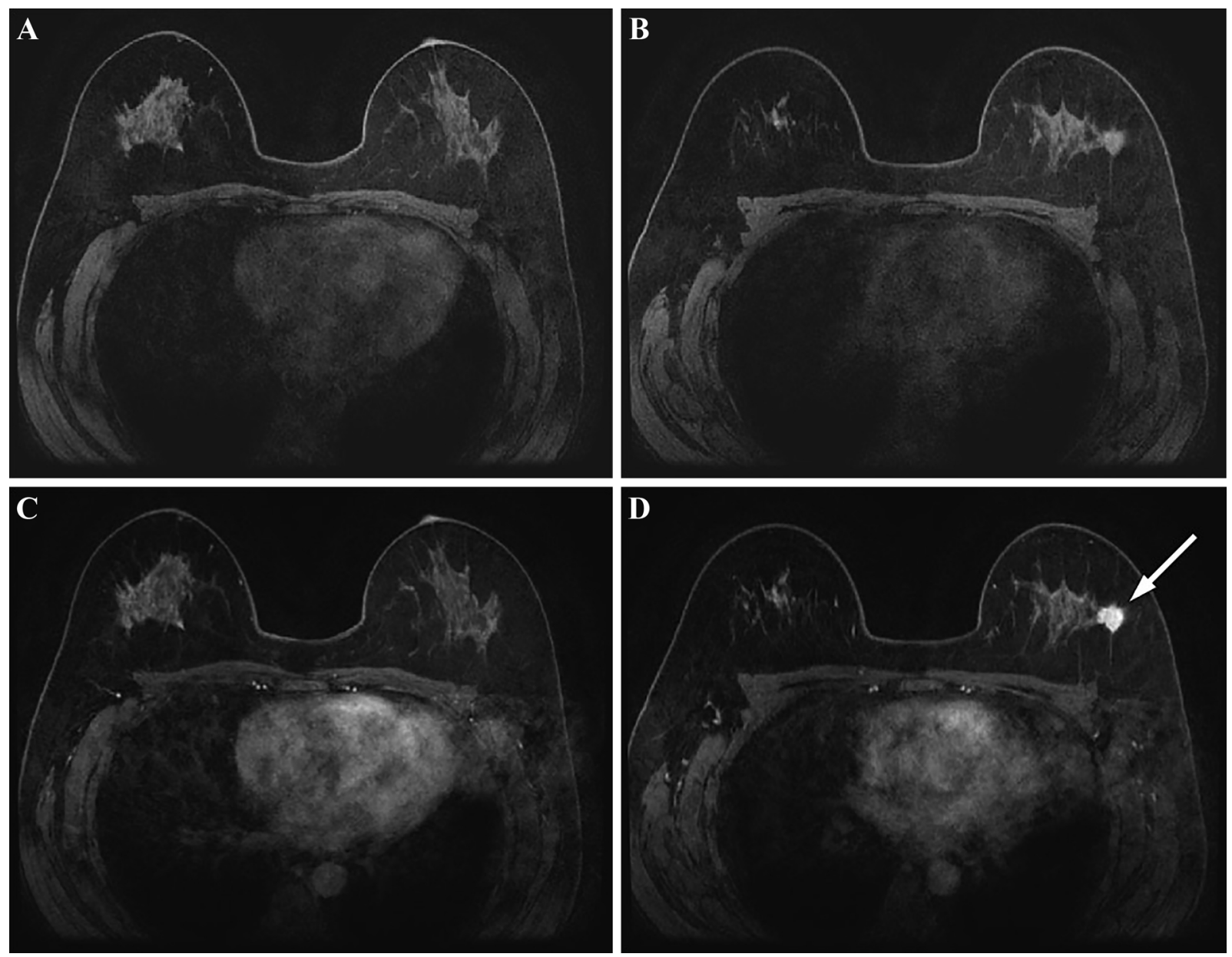

Fig. 4. A 47-year-old female with invasive ductal carcinoma at the proliferative phase.

An axial (a,b) pre-contrast and (c,d) post-contrast image clearly demonstrates an enhancing lesion in the outer area of the left breast (arrow). The BPE ratio was 0.24, and both observers classified the BPE as minimal. The C/B ratio was 15.4, and the qualitative cancer detectability was classified as excellent by both observers. 

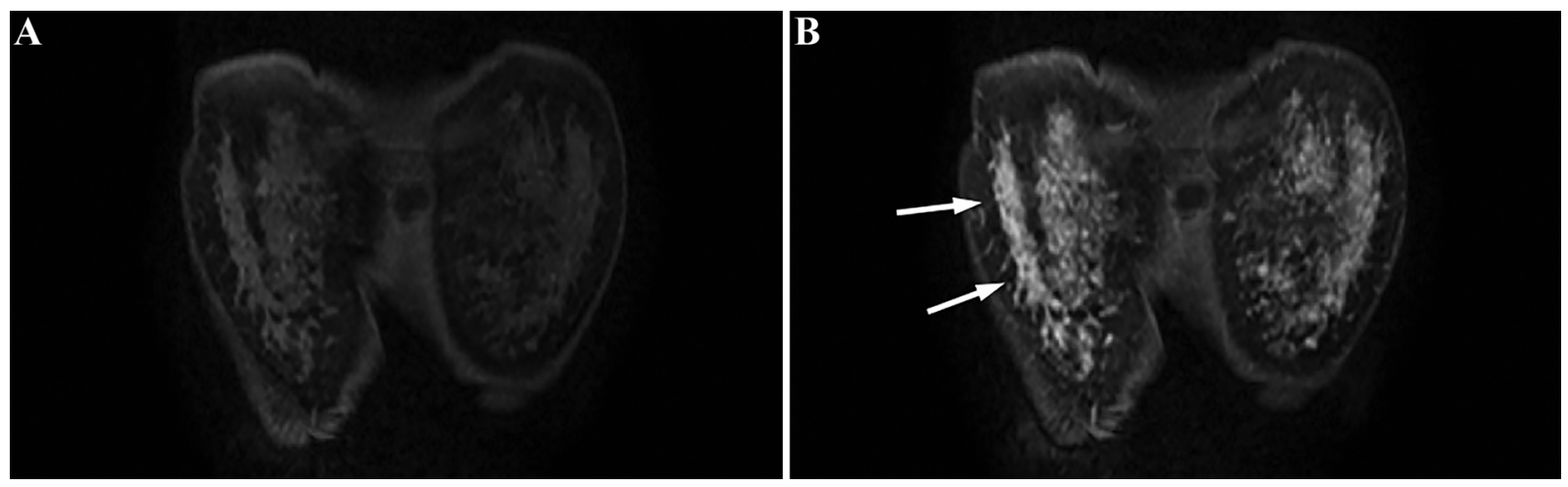

Fig. 5. A 48-year-old female with non-invasive ductal carcinoma at the secretory phase.

Though a coronal (a) pre-contrast and (b) post-contrast image demonstrates an enhancing lesion in the outer area of the right breast, it is obscured by the prominent BPE. The BPE ratio was 1.56, and both observers classified the BPE as marked. The C/B ratio was 1.2, and the qualitative cancer detectability was classified as poor by both observers.

enhancement was lowest in week 2 [12]. Muller-Schimpfle et al. reported that patients showed significantly lower parenchymal enhancement during days 7-20 of the menstrual cycle compared to days 21-26 [13]. Delille et al. showed that the maximum change in parenchymal enhancement occurred between days 3-7 and days 21-27, and they described days 3-14 of the menstrual cycle as the optimal time for dynamic contrast-enhanced breast MRI [11]. The guidelines issued by the European Society of Breast Imaging note that the optimal time to perform a breast MRI in premenopausal women is between the 5th and 12th day after the start of the menstrual cycle [6].

However, the results of our present analyses revealed that not only the proliferative phase but also the menstrual phase is suitable for breast MRI. We speculate that the difference in study populations is one of the causes of this discrepant finding, since most of the previous studies evaluated Western populations. To our knowledge, the study by Kajihara et al. is the only study of this issue in an Asian population, and it was conducted at a single center [14]. In their study, Kajihara et al. stated that the weakest BPE was shown on days 5-12 of the menstrual cycle. However, we note that their findings on days 1-4 were comparable to those on days 5-12. Our present findings suggest that the appropriate phase of the menstrual cycle for breast MRI in premenopausal Asian women might be longer than those in Western countries. This greater length of time can provide benefits for both patients and physicians.

Regarding the relationship between breast composition and BPE, Uematsu et al. reported a significant correlation between BPE and mammographic density [15]. Kang et al. indicated that the influence of the menstrual cycle on BPE varied according to the breast composition, and that the optimal time for breast MRI could be different between dense and fatty breasts [8]. They described that fatty breasts showed the highest BPE in the 4th week and the lowest BPE in the 2nd week of the menstrual cycle, whereas dense breasts showed the highest BPE in the 3rd week and the lowest BPE in the 4th week. Breast compositions on mammography are prone to vary among racial groups. In general, the breasts of Asian women are relatively small in size and are commonly characterized as dense or heterogeneously dense compared to those of Western women [16-19]. We observed herein that not only the proliferative phase but also the menstrual phase was suitable for breast MRI examination. We speculated that the difference in the distributions of breast composition between Asian women and Western women might have contributed to our finding that the menstrual phase (1 st week) is also suitable for breast MRI. The clarification of the differences between patients with and without prominent BPE and the factors affecting BPE other than the menstrual cycle is a challenge to be addressed in future studies.

There are some limitations in this study. First, we assessed the effect of the menstrual cycle on BPE and cancer detectability only; we did not evaluate the diagnostic performance for characterizing breast lesions or the extent of breast cancer, due to the small number of pathologically proven benign lesions and the unavailability of the precise MR-pathologic correlation due to the multicenter design of this study. Second, we could not evaluate the patients' breast composition on mammography, because this additional information could not be obtained, even though it is possible that the difference in breast composition between Asian women and Western women could have contributed to our findings.

\section{Conclusions}

In conclusion, the menstrual cycle affects both BPE and the detectability of breast cancer. The menstrual phase as well as the proliferative phase seem to be suitable for breast MRI examinations of premenopausal Asian women.

\section{Acknowledgement}

This work was supported by a grant from Bayer Yakuhin Ltd. (Osaka, Japan). Part of the statistical analyses was conducted by Kondo Photo Process Co. Ltd. (Osaka, Japan).

\section{References}

[1] T. Hata, H. Takahashi, K. Watanabe, et al., Magnetic resonance imaging for preoperative evaluation of breast cancer: a comparative study with mammography and ultrasonography, J. Am. Coll. Surg. 198 (2004) 190-197.

[2] H. Satake, K. Shimamoto, A. Sawaki, et al., Role of ultrasonography in the detection of intraductal spread of breast cancer: correlation with pathologic findings, mammography and MR imaging, Eur. Radiol. 10 (2000) 1726-1732.

[3] S. Komatsu, C.J. Lee, Y. Hosokawa, et al., Comparison of intraductal spread on dynamic contrast-enhanced MRI with clinicopathologic features in breast cancer, Jpn. J. Clin. Oncol. 34 (2004) 515-518.

[4] M. Van Goethem, K. Schelfout, E. Kersschot, et al., MR mammography is useful in the preoperative locoregional staging of breast carcinomas with extensive intraductal component, Eur. J. Radiol. 62 (2007) 273-282.

[5] R.L. Ellis, Optimal timing of breast MRI examinations for premenopausal women who do not have a normal menstrual cycle, Am. J. Roentgenol. AJR 193 (2009) 1738-1740.

[6] R.M. Mann, C.K. Kuhl, K. Kinkel, et al., Breast MRI: guidelines from the European Society of Breast Imaging, Eur. Radiol. 18 (2008) 1307-1318.

[7] A.M. Scaranelo, M.C. Carrillo, R. Fleming, et al., Pilot study of quantitative analysis of background enhancement on breast MR images: association with menstrual cycle and mammographic breast density, Radiology 267 (2013) 692-700.

[8] S.S. Kang, E.Y. Ko, B.K. Han, et al., Background parenchymal enhancement on breast MRI: influence of menstrual cycle and breast composition, JMRI 39 (2014) $526-534$.

[9] A.R. Amarosa, J. McKellop, A.P.K. Leite, et al., Evaluation of the kinetic properties of background parenchymal enhancement throughout the phases of the menstrual cycle, Radiology 268 (2013) 356-365.

[10] S. Shin, E.S. Ko, R.B. Kim, et al., Effect of menstrual cycle and menopausal status on apparent diffusion coefficient values and detectability of invasive ductal carcinoma on diffusion-weighted MRI, Breast Cancer Res. Treat. 149 (2015) 751-759.

[11] J.P. Delille, P.J. Slanetz, E.D. Yeh, et al., Physiologic changes in breast magnetic resonance imaging during the menstrual cycle: perfusion imaging, signal 
enhancement, and influence of the T1 relaxation time of breast tissue, Breast J. 11 (2005) 236-241.

[12] C.K. Kuhl, H.B. Bieling, J. Gieseke, et al., Healthy premenopausal breast parenchyma in dynamic contrast-enhanced MR imaging of the breast: normal contrast medium enhancement and cyclical-phase dependency, Radiology 203 (1997) 137-144.

[13] M. Muller-Schimpfle, K. Ohmenhauser, P. Stoll, et al., Menstrual cycle and age: influence on parenchymal contrast medium enhancement in MR imaging of the breast, Radiology 203 (1997) 145-149.

[14] M. Kajihara, M. Goto, Y. Hirayama, et al., Effect of the menstrual cycle on background parenchymal enhancement in breast MR imaging, Magn. Reson. Med. Sci. 12 (2013) 39-45.

[15] T. Uematsu, M. Kasami, J. Watanabe, Should breast MRI be performed with adjustment for the phase in patients' menstrual cycle? Correlation between mammographic density, age, and background enhancement on breast MRI without adjusting for the phase in patients' menstrual cycle, Eur. J. Radiol. 81 (2012) 1539-1542.

[16] Y. Takamoto, H. Tsunoda, M. Kikuchi, et al., Role of breast tomosynthesis in diagnosis of breast cancer for Japanese women, Asian Pac. J. Cancer Prev. 14 (2013) 3037-3040.

[17] G. Maskarinec, I. Pagano, Z. Chen, et al., Ethnic and geographic differences in mammographic density and their association with breast cancer incidence, Breast Cancer Res. Treat. 104 (2007) 47-56.

[18] G. Maskarinec, L. Meng, G. Ursin, Ethnic differences in mammographic densities, Int. J. Epidermiol. 30 (2001) 959-965.

[19] L.A. Habel, A.M. Capra, Oestreicher $\mathrm{N}$ et al. Mammographic density in a multiethnic cohort, Menopause 14 (2007) 891-899. 